\title{
Subsonic Boundary-Layer Wavefront Spectra for a Range of Reynolds Numbers
}

\author{
Adam E. Smith ${ }^{1}$, Stanislav Gordeyev ${ }^{2}$, \\ University of Notre Dame, Notre Dame, Indiana, 46556 \\ Theresa Saxton-Fox ${ }^{3}$, and Beverley McKeon ${ }^{4}$ \\ California Institute of Technology, Pasadena, California, 91125
}

\begin{abstract}
Aero-optic measurements of turbulent boundary layers were performed in wind tunnels at the University of Notre Dame and California Institute of Technology for heated walls at a range of Reynolds numbers. Temporally resolved measurements of wavefronts were collected at a range of Mach numbers between 0.03 and 0.4 and the range of $\operatorname{Re}_{\theta}$ between 1,700 and 20,000. Wavefront spectra for both heated and un-heated walls were extracted and compared to demonstrate that wall heating does not noticeably alter the shape of wavefront spectra in the boundary layer. The effect of Reynolds number on the normalized spectra was also presented, and an empirical spectral model was modified to account for Reynolds number dependence. Measurements of $O P D_{r m s}$ for heated walls were shown to be consistent with results from prior experiments, and a method of estimating $O P D_{r m s}$ and other boundary layer statistics from wavefront measurements of heated-wall boundary layers was demonstrated and discussed.
\end{abstract}

\section{Introduction}

$\mathrm{T}$ HE turbulent density fluctuations that are present in the region immediately around an aerodynamic vehicle have been shown to alter the local speed of light passing into and/or out of the aircraft through the turbulent region. This phenomenon, known as the aero-optic problem, is the result of the Gladstone-Dale relation which relates index-of-refraction, $n$, and density in air, $\rho$, via the Gladstone-Dale constant, $K_{G D}$ (which is approximately $2.27 \times 10^{-4} \mathrm{~m}^{3} / \mathrm{kg}$ in air for visible wavelengths of light),

$$
n(\vec{x}, t)-1=K_{G D} \rho(\vec{x}, t) \text {. }
$$

Electromagnetic waves passing through unsteady turbulent flows are unsteadily distorted by the spatially- and temporally-fluctuating density fields present along the optical path. Small disturbances to optical wavefronts in the near-field can result in significant reductions in time-averaged and instantaneous on-target intensity at points very far away from the source aircraft [1,2]. A reduction in on-target intensity poses a significant problem for the performance of airborne optical systems in directed energy, imaging, and free-space communications applications.

As planar wavefronts propagate through these unsteady density distributions, the effect of turbulent density fluctuations on the propagation of light can be quantified by defining the Optical Path Length $(O P L)$ as the integral of the index-of-refraction of a medium along the physical length traversed by a ray of light:

$$
O P L(x, y, t)=\int_{a}^{b} n(x, y, z, t) d z=\int_{a}^{b}\left[K_{G D} \rho(x, y, z, t)+1\right] d z,
$$

\footnotetext{
${ }^{1}$ Graduate Student, Department of Mechanical and Aerospace Engineering, Hessert Laboratory for Aerospace Research, Notre Dame, IN 46556, Student Member.

${ }^{2}$ Research Associate Professor, Department of Mechanical and Aerospace Engineering, Hessert Laboratory for Aerospace Research, Notre Dame, IN 46556, AIAA Associate Fellow.

${ }^{3}$ Graduate Student, Department of Mechanical and Civil Engineering, California Institute of Technology, Pasadena, CA 91125.

${ }^{4}$ Professor, Graduate Aerospace Laboratories, California Institute of Technology, Pasadena, CA 91125. AIAA Associate Fellow.
} 
where $z$ is the direction of beam propagation. The resulting deviation from the average $O P L$ can then be expressed as the Optical Path Difference $(O P D)$,

$$
O P D(\vec{x}, t)=O P L(\vec{x}, t)-\overline{O P L(\vec{x}, t)},
$$

where the overbar denotes spatial averaging. It can be shown that $O P D$ is in fact the conjugate of the zero-mean wavefront, $W(\vec{x}, t)=-O P D(\vec{x}, t)$.

While some of the earliest investigations of the effects of compressible, turbulent flow on the propagation of light can be traced back to the early 1950s [3], it was not 1969 until Sutton [4] introduced the most widely-referred to theoretical formulation for the aberrating effects of turbulent boundary layers based on turbulence statistics, using an approach that was based heavily on Tatarski's [5] treatment of electromagnetic waves propagated through the atmosphere. A simplified form of the resulting 'linking equation' from [1] relating turbulence quantities and levels of optical distortions is given as

$$
O P D_{r m s}^{2}=2 K_{G D}^{2} \int_{0}^{L} \rho_{r m s}^{2} \Lambda_{\rho}(y) d y,
$$

where $O P D_{r m s}$ is the root-mean-square of the $O P D, \rho_{r m s}(y)$ is the root-mean-square density fluctuation profile along the beam direction, and $\Lambda_{\rho}(y)$ is the density correlation length [1]. Utilizing the assumption set forth by Malley, et al. [6] that as turbulent structures convect their corresponding wavefront aberrations convect with them, Jumper and Fitzgerald [1] showed that Sutton's 'linking equation' result is equivalent to the formulation put forth in [3]. Recent CFD studies of the aero-optics of TBL by Wang \& Wang, where wavefront aberrations were computed by integrating through the computed density field, have also shown good agreement predictions from the linking equation [7].

Critical to the aero-optic characterization of the turbulent boundary layer and other aero-optically active flows was the introduction of high-bandwidth wavefront sensing devices that allow for time resolved characterization of wavefront aberrations, rather than only time-average measurements of levels of $O P D_{r m s}$. Malley, et al. [6] were the first to introduce a wavefront sensor capable of time-resolved measurements measuring the angle at which a smallaperture beam was deflected by turbulent flow as a function of time; $\phi(t)$. Using the assumption that wavefront aberrations and turbulent structures convect together at some convection velocity $U_{C}$, a 1-D streamwise 'slice' of $O P D$ could be reconstructed by first computing the $O P L$ via the integral

$$
O P L(t)=-U_{C} \int_{0}^{t} \theta(\tau) d \tau,
$$

where $\tau$ is a placeholder for time. $O P D$ is then computed using equation (3).

Using the Malley probe, detailed measurements of wavefront spectra and convective speeds of aero-optical structures in subsonic boundary layers were collected by Gordeyev, et al [10], Wittich, et al. [11] and Cress [12]. The authors proposed a statistical scaling for $O P D_{r m s}$ for light traversing subsonic, compressible turbulent boundary layers in the wall normal direction that was consistent with experimental data,

$$
O P D_{r m s}=A \frac{\rho_{\infty}}{\rho_{S L}} \delta^{*} M_{\infty}^{2}
$$

where $\delta^{*}$ is the boundary layer displacement thickness, and the empirical constant of proportionality $A=$ $(1.7 \pm 0.2) \times 10^{-5}$. The authors found that measurements of convective velocity of aero-optical structures in TBL were found to be about $U_{C}=0.82 U_{\infty}$. This result, and the observation that the peak of measured deflection angle spectra is located around $S t_{\delta}=1$, suggests that the most optically active structures reside in the outer region of the turbulent boundary layer. These experimental findings have been supported by recent computational investigations of the aero-optics of boundary layers by Wang \& Wang [7]. Recent careful comparisons of 1-D high-bandwidth wavefront measurements made with the Malley probe, and spatially and temporally resolved wavefront measurements made with the high-speed Shack-Hartmann wavefront sensor have shown good agreement for turbulent boundary layers in the transonic $\left(M_{\infty}=0.4 . .0 .7\right)$ regime, validating the accuracy of the underlying assumptions of the Malley probe wavefront reconstruction for TBL measurements [9].

Wyckham \& Smits [8] pioneered the use of a high-speed camera and a lenslet array to create a high-bandwidth Shack-Hartmann wavefront sensor, which allowed for measurements of aero-optic aberrations with good spatial and temporal resolution. Using a bulk-flow analysis they derived the more-general scaling law for $O P D_{r m s}$ including supersonic regime as $O P D_{r m s} \sim \rho M^{2} \delta \sqrt{C_{f}} r_{2}^{-3 / 2}, \quad r_{2}=1+\frac{\gamma-1}{2} M^{2}\left[1-r\left(U_{C} / U_{\infty}\right)^{2}\right]$. Based on aero-optical 
measurements for a supersonic boundary layer at $M=2.0$ and using the linking equation, Gordeyev, et al. [13, 14] derived an alternative general scaling law for $O P D_{r m s}$,

$$
O P D_{r m s}=0.2 K_{G D} \rho \delta M^{2} \sqrt{C_{f}} G(M),
$$

where $G(M)=1-0.19 M^{2}+0.03 M^{4}$ for $M<1.5$ and showed that this scaling law give very similar prediction compared to the scaling law by Wyckham \& Smits [8]. Since for $M<0.4,1>G(M)>0.97$, the scaling may be simplified by neglecting the $G(M)$ term for the remainder of this paper.

Additional experimental and modeling work has been performed by Cress, et al. [15] in characterizing the aerooptic effects of turbulent boundary layers as a function of the wall temperature, $T_{w}$. Using the Extended Strong Reynolds Analogy (ESRA), scaling relationships were developed and shown to be consistent with experimental data [15]:

$$
O P D_{r m s}=0.2 K_{G D} \rho_{\infty} \delta \sqrt{C_{f}}\left[M^{4}+C_{1}\left(\frac{\Delta T}{T_{\infty}}\right) M^{2}+C_{2}\left(\frac{\Delta T}{T_{\infty}}\right)^{2}\right]^{\frac{1}{2}}
$$

where $\Delta T=T_{w}-T_{a w}$, the difference between wall temperature $T_{w}$ and the adiabatic wall temperature, $T_{\infty}$ is the freestream total temperature, and $C_{1}$ and $C_{2}$ are constants that can be determined from TBL velocity profiles or wavefront measurements. A linearized form of equation (8) was found to work well for modeling wavefront measurements for wall-heating,

$$
O P D_{r m s}=0.2 K_{G D} \rho_{\infty} \delta \sqrt{C_{f}}\left[M^{2}+D_{1}\left(\frac{\Delta T}{T_{\infty}}\right)\right],
$$

where $D_{1}=C_{1} / 2$ [15]. Likewise, single boundary layer deflection angle spectra for heated wall boundary layers were shown to scale well with the linearized form of equation (8):

$$
\hat{\phi}_{N O R M}^{p k}=\hat{\phi}^{p k} /\left[\rho_{\infty} \delta \sqrt{C_{f}}\left(M^{2}+D_{1}\left(\frac{\Delta T}{T_{\infty}}\right)\right)\right] .
$$

Note that in equations (9) and (10), the heated wall temperature for aero-optic wavefront measurements works as a linear amplification of the baseline spectra, with no resulting shift in peak frequency or spectra shape. Then for low subsonic freestream velocities and thin boundary layer thicknesses which have a weak aero-optic signature, it is possible to use wall heating as a passive 'marker' to make low-Reynolds number TBLs aero-optically 'visible'. Thus wavefront sensors can be used as non-intrusive diagnostic tools to allow for investigation of the dynamics of turbulent boundary layers at relatively low Reynolds numbers. Also, the heating technique can provide valuable experimental wavefront information at low Reynolds numbers, both to verify/correct various scaling models at low Reynolds numbers and to provide a direct comparison with computational simulations, as currently there is a large Reynolds number "gap" between experimental measurements with typical $\operatorname{Re}_{\theta}>20,000$ and numerical simulations $[7,16]$ with much lower $\operatorname{Re}_{\theta} \sim 1,000$ to 3,000 .

\section{Experimental Setup}

One set of measurements was conducted in the Hessert Transonic Wind Tunnel (TWT) at the University of Notre Dame. The Transonic Wind Tunnel, shown in Figure 1, is a continuous flow indraft wind tunnel with an inlet contraction ratio of $150: 1$, and a cross-section of $10 \mathrm{~cm} \times 9.9 \mathrm{~cm}$ in the tunnel test section, which is constructed of Plexiglas. The test section length can be varied by adding or removing portions of the Plexiglas boundary layer development section. The TWT is able to operate at a range of freestream Mach numbers from 0.18 to 0.70 . The total length of the boundary layer development section is variable, and can be lengthened or shortened using $0.3 \mathrm{~m}$ modular sections to change the boundary layer thickness at the measurement section. In the current study, the total length to the beginning of the optical section is approximately $100 \mathrm{~cm}$.

Non-adiabatic wall temperatures were introduced by replacing the Plexiglas wall with an aluminum wall with an $8 \mathrm{~mm}$ thick Aluminum plate for the first $100 \mathrm{~cm}$ of the test section, and heating it from the outside surface using strips of flexible electric resistive coil heaters, similar to the experimental configuration described in [15]. Just downstream of the boundary layer development/wall heating section, portions of the Plexiglas on the upper and lower walls of the wind tunnel were replaced with optical quality glass plates to allow accurate optical characterization of the boundary layer using aero-optic wavefront sensors. A Pitot tube mounted downstream of the optical measurement location was used to measure the free-stream velocity throughout the experiment. 


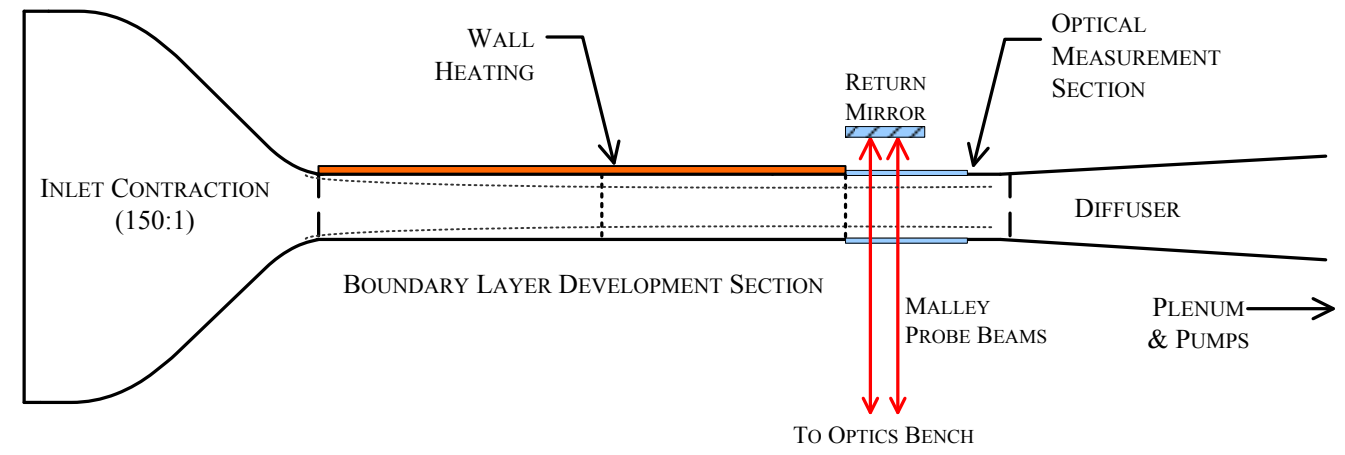

Figure 1. Schematic of the Hessert Transonic Wind Tunnel (TWT) configured for Malley probe wavefront measurements of aero-optic aberrations from heated wall turbulent boundary layers.

Experiments at California Institute of Technology were performed in the Merrill Wind tunnel, a recirculating tunnel with a $0.6 \times 0.6 \times 2.4 \mathrm{~m}$ constant area test section, pictured in Figure 2 . The wall of a test plate mounted in the center of the test section was heated at two streamwise locations using the same flexible heating panels as in the Notre Dame experiments, this time sandwiched between the aluminum test surface and a heavily insulated lower surface. The wall temperature on the measurement side was held constant at $43^{\circ} \mathrm{C}$, as determined by surfacemounted thermocouples.

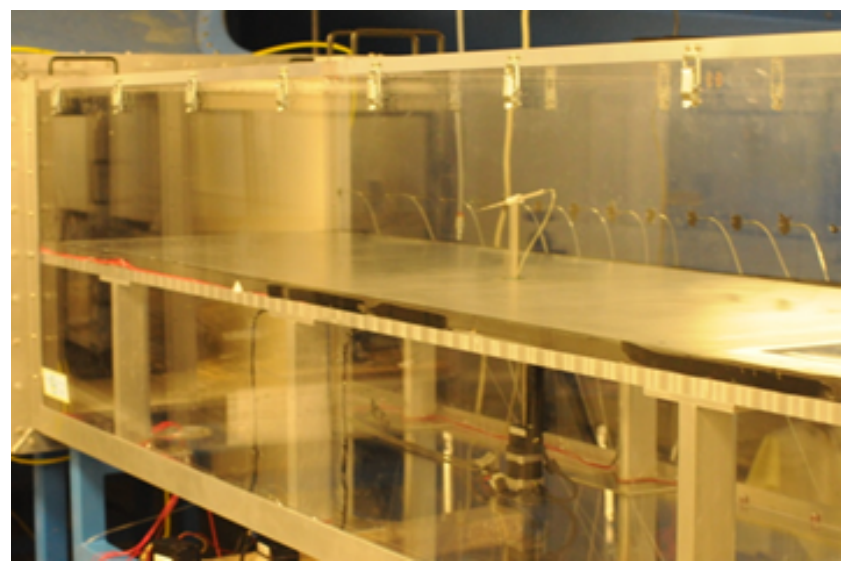

Figure 2. Photograph of the Merrill Wind Tunnel flat plate test section. Flow is from left to right.
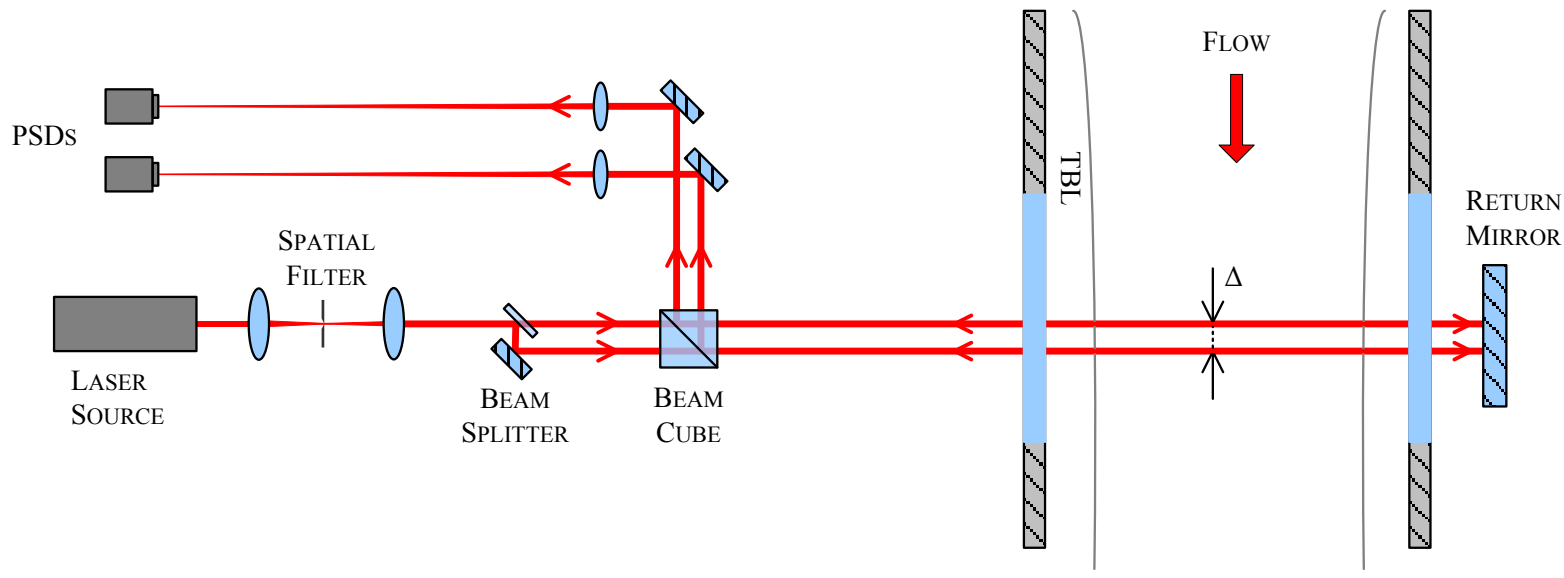

Figure 3. Schematic of the Malley Probe wavefront sensor. 
Wavefront measurements were acquired downstream of the heated wall boundary layer development section in both facilities using the Malley Probe 1-D wavefront sensor, which is shown schematically in Figure 3 and is described in detail by Gordeyev, et al [13]. Malley probe data were acquired in the Notre Dame TWT at a streamwise location of $105 \mathrm{~cm}$ at freestream velocities $V_{\infty}=65$ to $140 \mathrm{~m} / \mathrm{s}$. In the Caltech MWT data were collected at a freestream velocity of $9.4 \mathrm{~m} / \mathrm{s}$ and a Malley probe was positioned $5 \mathrm{~cm}$ downstream of the end of the first heating plate, $1.05 \mathrm{~m}$ from the leading edge and $1 \mathrm{~m}$ from the estimated virtual origin of the (tripped) turbulent boundary layer. The Reynolds number at the location of the Malley probe was $\operatorname{Re}_{\theta} \sim 1700$, based on previous measurements at this location using a hot wire. The Mach number $M$, boundary layer thickness $\delta$, Reynolds number $\operatorname{Re}_{\theta}$, wall temperature $\Delta T$, and sampling rate $f_{\text {samp }}$, corresponding to each of these cases are given in Table 1 .

From the time series of beam deflection angle $\phi(t)$ obtained with the Malley probe, a number of statistical quantities, including deflection angle amplitude spectra, one-dimensional wavefronts, and $O P D_{r m s}$ were computed using the frozen-flow assumption, $d x=U_{C} d t$, where the convective velocity $U_{C}$ is computed from the phase delay between readings from two Malley beams aligned in the streamwise direction with separation $\Delta$.

Table 1. Description of TBL conditions for heated-wall Malley probe wavefront measurements.

\begin{tabular}{ccccccc} 
Facility & $\boldsymbol{V}_{\infty}[\mathbf{m} / \mathbf{s}]$ & $\boldsymbol{M}$ & $\boldsymbol{\delta}[\mathbf{c m}]$ & $\mathbf{R e}_{\boldsymbol{\theta}}$ & $\boldsymbol{\Delta} \boldsymbol{T}[\mathbf{K}]$ & $\boldsymbol{f}_{\text {samp }}[\mathbf{k H z}]$ \\
\hline Caltech MWT & 9.4 & 0.03 & 2.7 & 1,700 & 21 & 30 \\
ND TWT & 64.8 & 0.18 & 1.2 & 4,200 & $15-28$ & 200 \\
$"$ & 98.8 & 0.28 & 1.2 & 5,700 & $9-24$ & 200 \\
$"$ & 118 & 0.35 & 1.2 & 7,900 & $7-28$ & 200 \\
$"$ & 140 & 0.41 & 1.2 & 9,000 & $7-24$ & 200 \\
$"$ & 140 & 0.41 & 2.4 & 20,000 & 0 & 200 \\
\hline \hline
\end{tabular}

To remove the aero-optic contribution of the, un-heated TBL along the lower wall in the TWT facility in the Malley probe wavefront measurements, boundary layers on opposite walls of the test section are assumed to be statistically independent. Then the contribution of the heated wall single boundary layer (SBL), $O P D_{r m s}^{\mathrm{SBL}, T_{w}}$ may be isolated from the contribution of the un-modified boundary layer using an extension of the statistical relationship shown in [11,14], $O P D_{r m s}^{\mathrm{SBL}, T_{w}}=\sqrt{\left(O P D_{r m s}^{\mathrm{DBL}}\right)^{2}-\frac{1}{2}\left(O P D_{r m s}^{\mathrm{BASELINE}}\right)^{2}}$, where $O P D_{r m s}^{\mathrm{DBL}}$ is the value of $O P D_{r m s}$ measured by a wavefront sensor in the double boundary layer (DBL) configuration, in which the beams pass through both the wall-heating modified boundary layer and the un-modified boundary layer. Likewise $O P D_{r m s}^{\text {BASELINE }}$ is the DBL measured value of $O P D_{r m s}$ for two un-modified boundary layers in the control case. Similarly, it is also shown in [15] that deflection angle spectra of the LEBU-modified boundary layer can be extracted in a similar manner; $\hat{\phi}_{S B L, T_{w}}=\sqrt{\hat{\phi}_{D B L}^{2}+0.5 \hat{\phi}_{B A S E L I N E}^{2}}$. From the SBL scaled deflection angle spectra obtained for different wall temperatures, $O P D_{r m s}$ were computed using the Fourier-version of equation (6) and plotted as a function of recovery temperature $\Delta T$. For all cases, this method of computing $O P D_{r m s}$ allows for the exclusion of both low-frequency noise from tunnel vibration, and high-frequency noise peaks that are the result of RF and electronic interference sources present in and around the laboratory environment.

\section{Results \& Discussion}

Malley probe wavefront measurements were obtained for the conditions described previously at the Caltech and Notre Dame wind tunnel facilities, and a number of statistics, including deflection angle amplitude spectra and convective velocity were computed and analyzed. For both the Caltech wavefront data obtained at $\operatorname{Re}_{\theta}=1,700$ the convective velocity was found to be $0.83 U_{\infty}$ using the correlation of the two beams, a value which is consistent with wavefront measurements obtained previously at higher Reynolds numbers [9,14]. For wavefronts obtained in the Notre Dame facility, the convective velocity was found to be $0.82 U_{\infty}$, which is also consistent with previous measurements in this facility at higher Reynolds numbers. This independence of these results with respect to $\operatorname{Re}_{\theta}$ suggests that the most aero-optically influential components of the TBL are large-scale structures located in the outer region of the boundary layer, since changes in Reynolds number would correspond to changes in the inertial sub-range near the wall.

To investigate the effect of changes in Reynolds number on the deflection angle amplitude spectra measured with the Malley probe, heated wall deflection angle spectra were normalized using the relationship from Cress [15] shown in equation (9), with $D_{1}$ being computed from experimental data in the same manner described in [15]. 
However, the scaling relationship given in (9) has to date only been shown experimentally to be valid for Reynolds numbers as low as 9,000 [15], while the majority of the data in the present study has been obtained at Reynolds numbers that are substantially lower. The validity of the scaling, however, can be quickly checked by investigating the collapse of deflection angle spectra using equation (9) for a number of wall-heating temperature values at each Reynolds number. The results of this investigation are shown in Figures 4 and 5 for $\operatorname{Re}_{\theta}=4,200$ and 9,000 respectively.
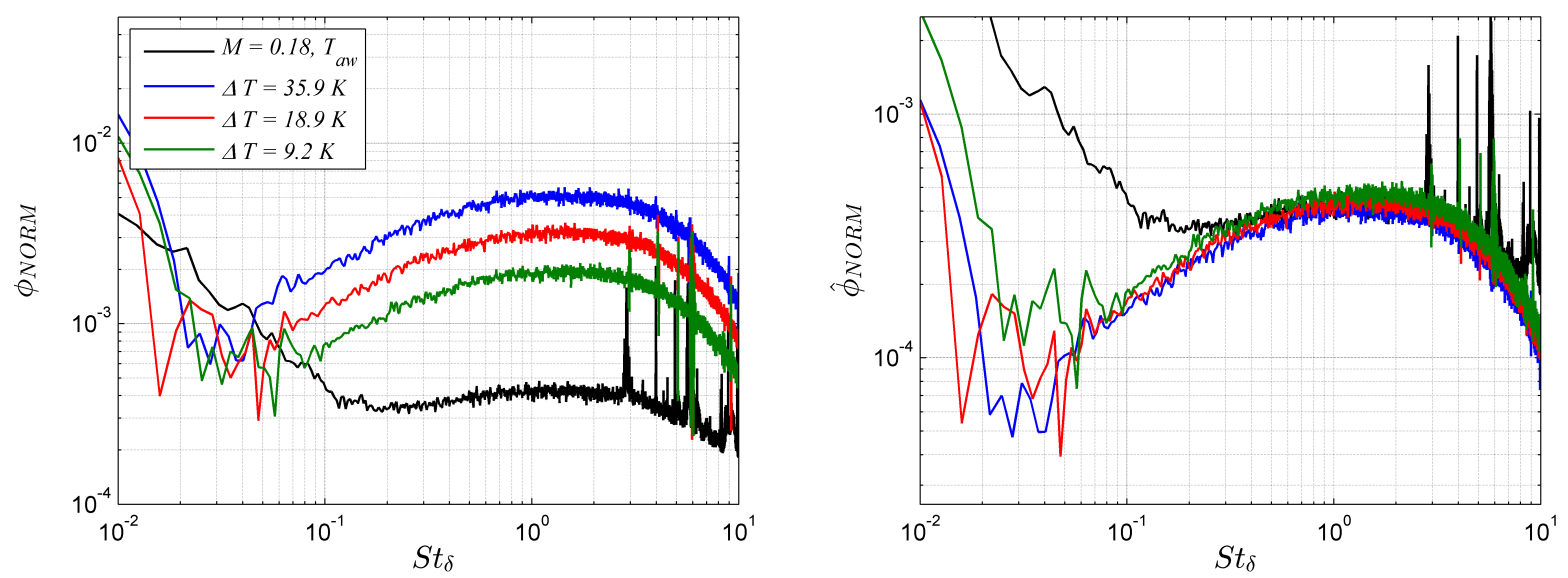

Figure 4. $\operatorname{Re} \theta=4,200(M=0.18)$ deflection angle spectra, normalized by equation (9) with $D_{1}=0$ (left) and $D_{1}$ computed from Malley probe wavefront data (right).
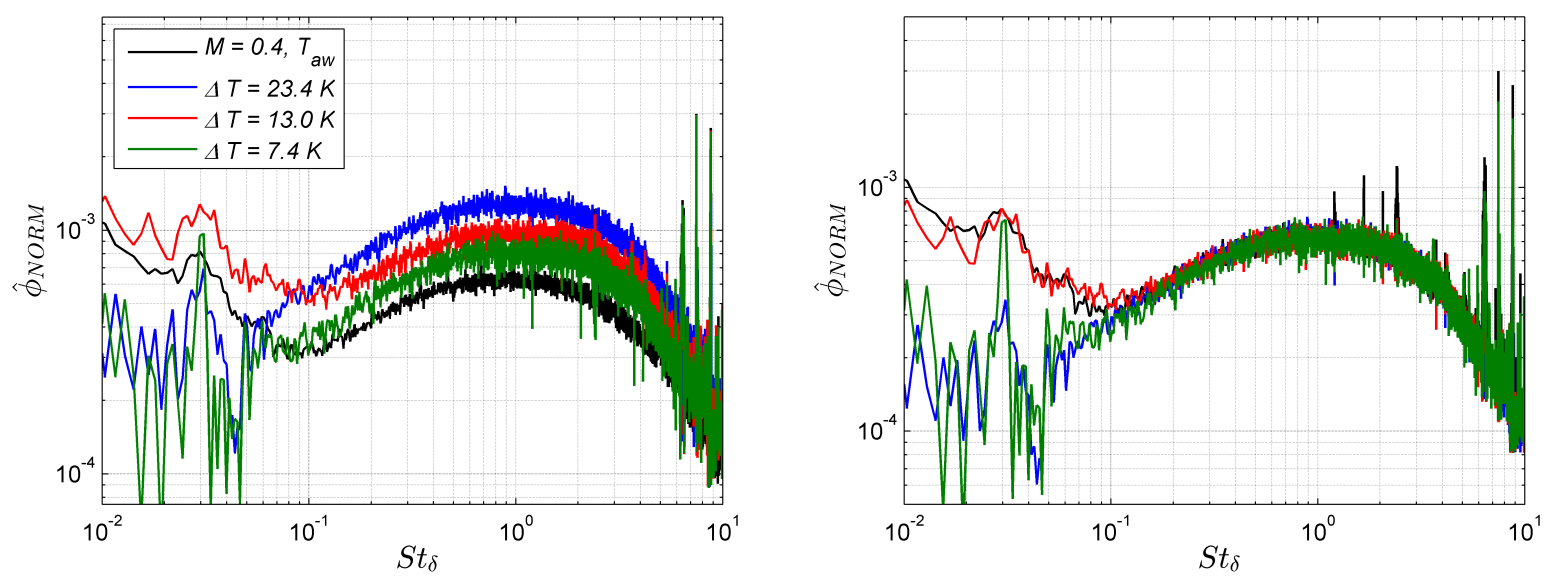

Figure 5. $\operatorname{Re} \theta=9,000(M=0.4)$ deflection angle spectra, normalized by equation (9) with $D_{1}=0$ (left) and $D_{1}$ computed from Malley probe wavefront data (right).

For both Figure 4 and 5, good self-similarity is observed when normalizing the deflection angle spectra for a large range of wall temperatures by equation (9) using $D_{1}$ computed from the data. This confirms that the use of wall heating as a passive amplifier of boundary layer turbulence for aero-optic wavefront measurements does not noticeably alter the wall-normal integrated characteristics of density fluctuations in the TBL. Therefore, heated-wall deflection angle spectra can be measured and normalized in this way in order to compare spectra obtained at low Reynolds numbers, circumventing the problem of aero-optic 'invisibility' that occurs when freestream velocities enter the incompressible regime.

\section{A. Effect of Reynolds number on wavefront spectra}

Figure 6 presents normalized deflection angle spectra obtained using wall heating as a passive amplifier for the different Reynolds number conditions described in Table 1. In the vicinity of the spectra peak, and for the low end of the spectral peak $\left(S t_{\delta}<1\right)$, all cases show good collapse. The peaks for all measured spectra are at $S t_{\delta}=1.0$, independent of Reynolds number. In [14] it was shown that using Kolmogorov-like arguments for the inertial range, the deflection angle amplitude spectrum at large frequencies should behave as $\sim f^{(-2 / 3)}$. This spectra behavior is 
plotted in Figure 6 as a dashed black line. The spectrum at the lowest Reynolds number quickly falls off of this theoretically predicted behavior, indicating a fairly small inertial range, while the fall-off is less drastic for larger Reynolds numbers, implying a larger inertial range.

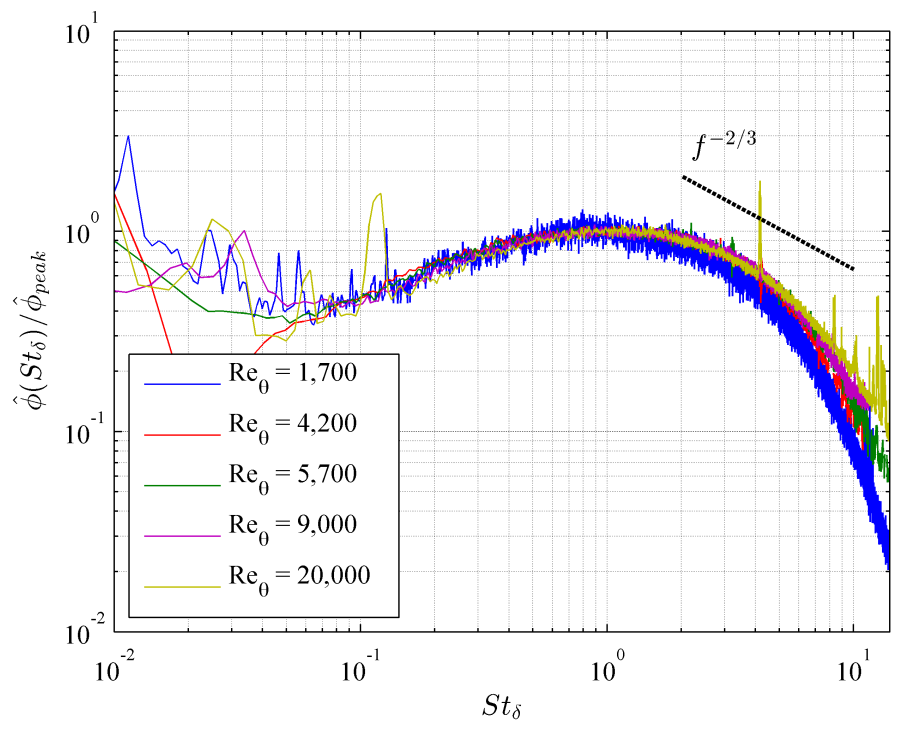

Figure 6. Normalized deflection angle spectra for different Reynolds numbers.

To model the observed changes in the (high frequency slope) size of the inertial range of deflection angle spectra for different Reynolds numbers, the spectral model from [14] was modified in order to account for changes in the spectra roll-off as a function of $\operatorname{Re}_{\theta}$. The fall-off of the spectra was assumed to take a form inspired by Tatarski's modification of Kolmogorov's atmospheric wavefront spectrum to account for the presence of inner scale dissipative structures [17]. Seeking a function in the form of the original model times the exponential term, the modified spectral model was found to be

$$
\hat{\phi}_{f i t}\left(S t_{\delta}\right)=\hat{\phi}_{\text {peak }} \frac{S t_{\delta}}{1+\left(S t_{\delta} / 0.83\right)^{5 / 3}} \exp \left[-\left(\frac{S t_{\delta}}{f\left(\operatorname{Re}_{\theta}\right)}\right)^{2}\right] .
$$

The $f\left(\operatorname{Re}_{\theta}\right)$ term was empirically determined to be $f\left(\operatorname{Re}_{\theta}\right) \approx 1.6 \operatorname{Re}_{\theta}{ }^{(0.22)}$ by fitting equation (11) to the experimental data presented in Figure 6.

Figure 7 shows comparisons between experimental data, the original TBL spectral model from [14], and the modified model for four Reynolds numbers ranging from 1,700 to 20,000. For $S t_{\delta}<0.5$, the experimentally measured spectra and model spectra deviate from one another, with the experimentally measured spectra containing more energy. This behavior is consistent with results from [14], where it was shown that for Malley probe measurements, this increase in energy is a result of contamination from mechanical vibrations in Malley probe data that cannot be properly removed [14]. For $S t_{\delta}>0.5$, Figure (7) shows that the modified spectral model given in equation (11) does a good job of describing the high-frequency roll-off behavior for a large range of Reynolds numbers from both the Caltech and Notre Dame facilities.

Knowing the deflection angle spectra, several important statistical properties of the boundary layer can be computed: overall levels of $O P D_{r m s}$, the aperture function, $G(A p, \delta)$, and the cross-correlation function, $R(\Delta x / A p)$ [14]:

$$
\begin{gathered}
O P D_{r m s}^{2}=2 U_{c} \int_{0}^{\infty} \frac{|\hat{\phi}(f)|^{2}}{(2 \pi f)^{2}} d f \\
G(A p / \delta) \equiv O P D_{r m s}(A p) / O P D_{r m s}, \text { where } O P D_{r m s}^{2}(A p)=2 U_{c} \int_{0}^{\infty} A F(A p, f) \frac{|\hat{\phi}(f)|^{2}}{(2 \pi f)^{2}} d f, \\
R(\Delta x / A p ; A p)=\int_{0}^{\infty} K\left(f A p / U_{\infty}, \Delta x / A p\right) \frac{|\hat{\phi}(f)|^{2}}{(2 \pi f)^{2}} d f .
\end{gathered}
$$


The transfer functions $A F$ and $K$ are given in [14].
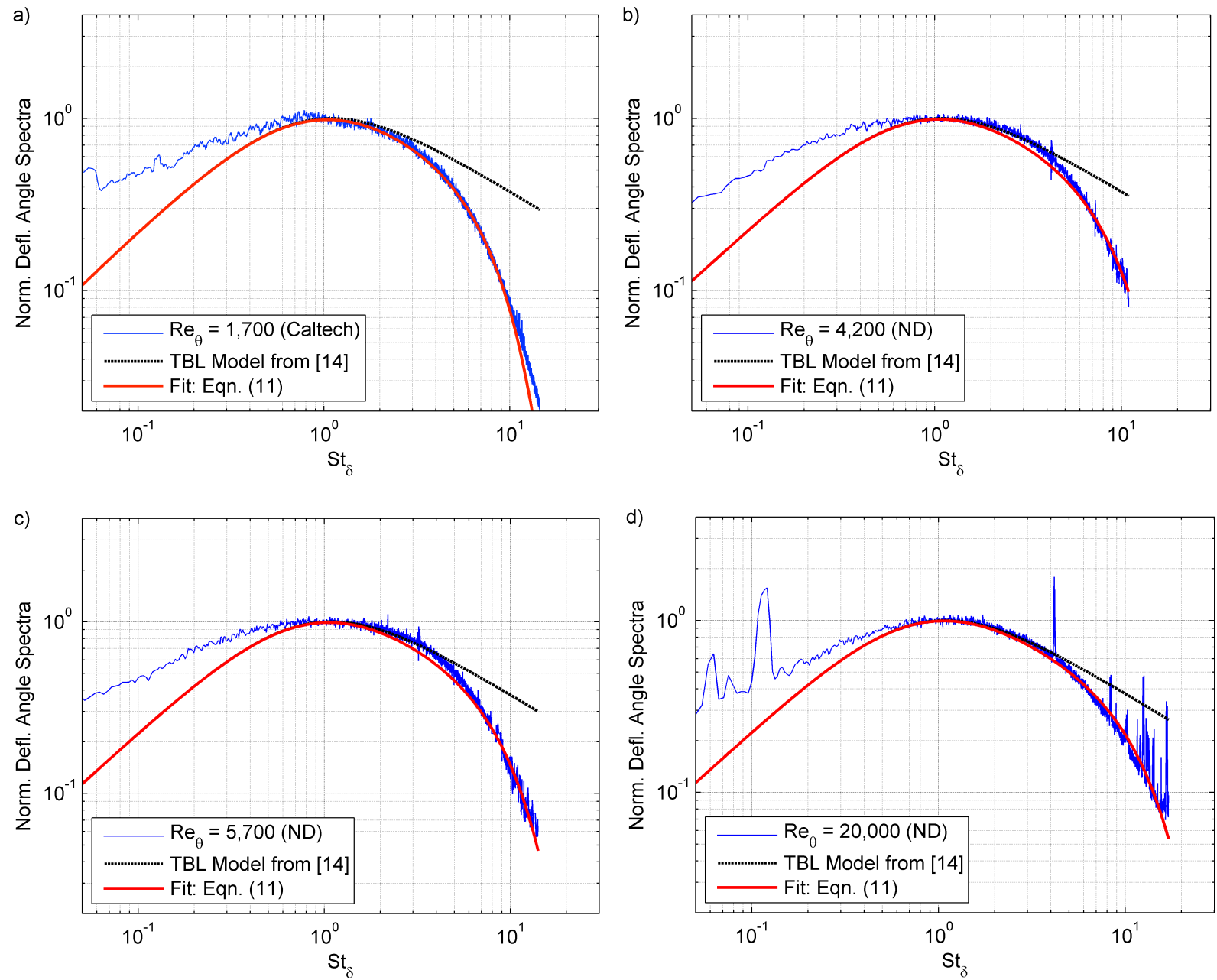

Figure 7. Comparison of experimental deflection angle spectra and the modified empirical model from equation (11) for Reynolds number a) 1,700 (Caltech), b) 4,200, c) 5,700, and d) 20,000 (ND).

\section{B. Calculation of $O P D_{r m s}(\Delta T=0)$ from heated wall data}

From equation (12a), the levels of $O P D_{r m s}$ were computed from SBL scaled deflection angle spectra at different wall heating temperatures for $\operatorname{Re}_{\theta}=4,200-9,000$. Figure 8 presents these data as a function of $\Delta T$, and it is apparent that $O P D_{r m s}$ increases proportionally with wall temperature, consistent with the corresponding wall heating model given in equation (9) $[12,14]$.

If $M^{2}$ is pulled out of the bracketed term, equation (9) becomes

$$
O P D_{r m s}=0.2 K_{G D} \rho_{\infty} \delta M^{2} \sqrt{C_{f}}\left[1+D_{1}\left(\frac{\Delta T}{T_{\infty} M^{2}}\right)\right] \text {. }
$$

For the case of $\Delta T=0$ (no wall heating), the bracketed term is equal to unity and $O P D_{r m s}^{(\Delta T=0)}=B K_{G D} \rho_{\infty} \delta M^{2} \sqrt{C_{f}}$ is recovered for the un-heated wall. Substituting this back in to equation (13) and expanding,

$$
O P D_{r m s}(\Delta T)=O P D_{r m s}^{(\Delta T=0)}+\frac{D_{1} O P D_{r m s}^{(\Delta T=0)}}{T_{\infty} M^{2}}(\Delta T) .
$$

Using equation (14), measurements of wavefront aberrations for heated walls at several wall temperatures may be used to recover estimates of $O P D_{r m s}^{(\Delta T=0)}$ by obtaining the $y$-intercept value from a linear regression fit on the heated wall $O P D_{r m s}$ versus $\triangle T$ data. This technique allows one to reliably measure aero-optical properties of turbulent 
boundary layers at low Reynolds numbers or, equivalently, for thin boundary layers at low speeds, where direct measurements of un-heated boundary layers might result in fairly large error, as the aero-optical signal for low Re is very weak. Assuming a relative error in heated-BL $O P D_{r m s}$ measurements of approximately $\pm 10 \%$ (from the measurement precision of $\pm 8 \%$ of Malley probe beam spacing, and therefore $U_{C}$ ), and an absolute error in temperature measurement of $\pm 1 \mathrm{~K}$, a linear regression was performed on the heated wall data presented in Figure (8) for each Reynolds number using the method from York, et al. [18], which also returns error bars on the $y$ intercept.

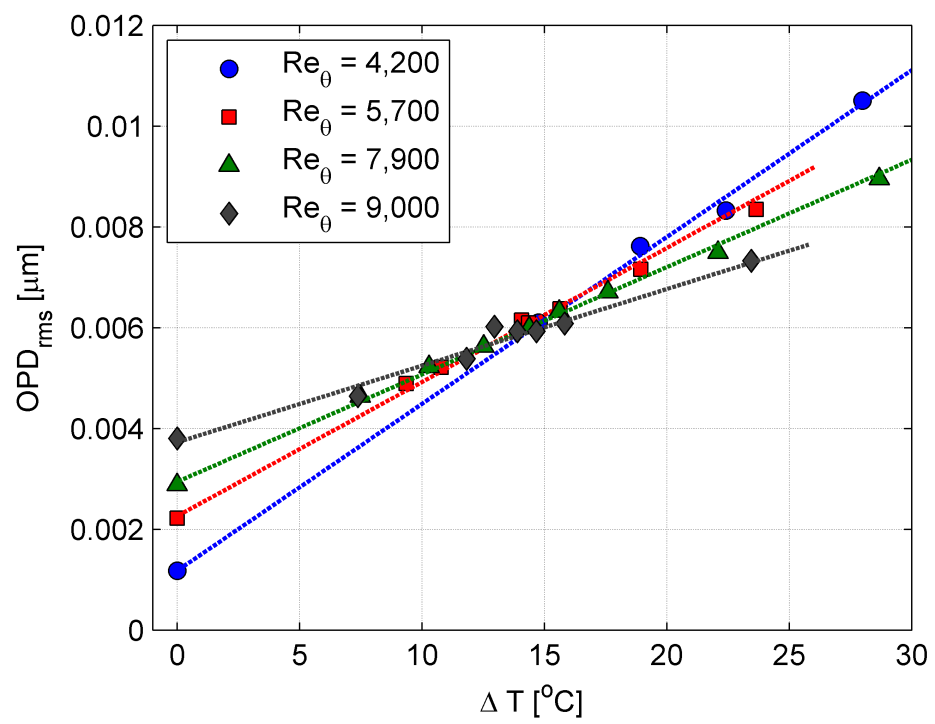

Figure 8. Levels of wavefront aberrations as a function of wall temperature change for a range of low Reynolds numbers.

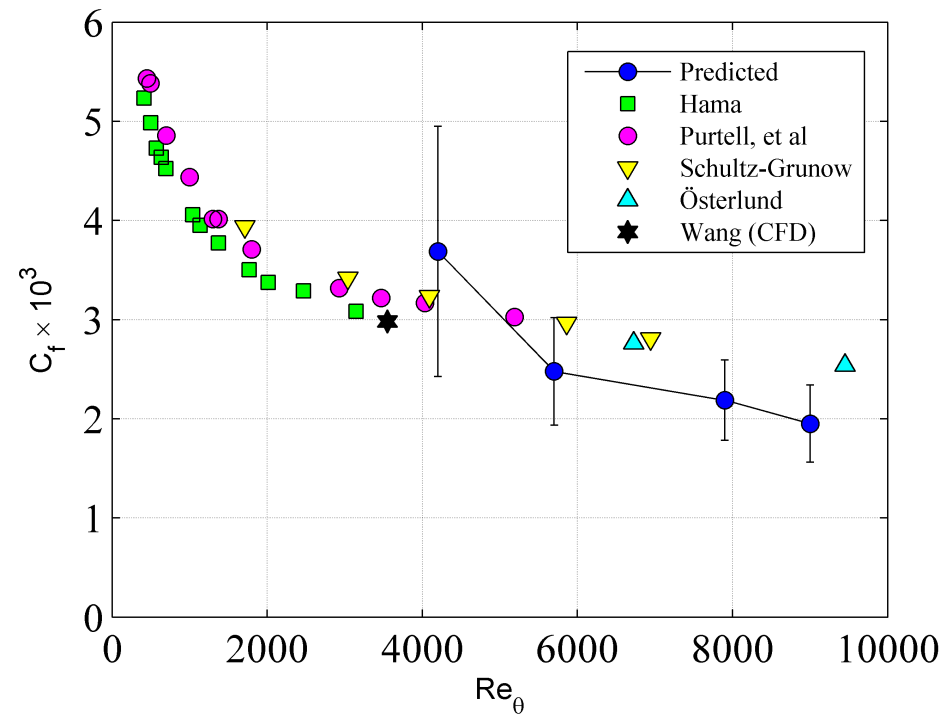

Figure 9. Estimates of skin friction coefficient, $C_{f}$, for low-Reynolds number TBL from levels of $O P D_{r m s}$ compared with measured values from other experimental studies.

One way to check the validity of the scaling, equation (7), at low Reynolds numbers is to recognize that $C_{f}$ is the only term which explicitly depends on $\operatorname{Re}_{\theta}$, while the rest of them, $\rho, \delta, M$, are not. Rearranging equation (7) for $C_{f}$ gives,

$$
C_{f}=\left[\frac{O P D_{r m s}^{(\Delta T=0)}}{0.2 K_{G D} \rho_{\infty} \delta M^{2} G(M)}\right]^{2} .
$$


If all of the assumption used to derive equation (7), namely the Strong Reynolds Analogy and the mixed scaling for the mean and fluctuating velocity profiles [14], are valid, the $C_{f}$-estimation based on $O P D_{r m s}$, equation (15), should predict the same value for $C_{f}$ as from direct measurements. Let us emphasize that equation (15) is not about measuring $C_{f}$, but rather testing the validity of the scaling, equation (7), at low Reynolds numbers, as it was originally derived for high Reynolds number TBLs. Any deviation between "optical" $C_{f}$, computed from equation (15), and true $C_{f}$ simply indicates that the scaling assumptions are not valid at this Reynolds number. Figure 9 shows estimated values of $C_{f}$, with error bars, from the $O P D_{r m s}$ measurements; Figure 9 demonstrates that values of skinfriction "computed" from heated-wall wavefront measurements are consistent with experimental measurements of $C_{f}$ from Hama [19], Purtell, et al. [20], Schultz-Grunow [21], and Österlund [22] in the low-Reynolds number range. From here we concluded that the prediction for $O P D_{r m s}$, equation (7), should be valid for $\operatorname{Re}_{\theta}>4,000$. Figure 9 also shows an estimated value of $C_{f}$ "computed" from $O P D_{r m s}$ obtained from optical simulations by Wang and Wang at $\operatorname{Re}_{\theta}=3,550$ [7]. It was found that there was less than $1 \%$ difference between $C_{f}$ computed from equation (15) and the value computed directly from CFD, and Figure 9 shows that these values are also consistent with experimental measurements.

\section{Conclusions}

Results of aero-optic wavefront measurement of heated-wall turbulent boundary layers for a range of subsonic Mach numbers ( 0.03 to 0.4 ) and Reynolds numbers, $\operatorname{Re}_{\theta}=1,700$ to 20,000 , are presented. Data were collected independently in two facilities: the Transonic Wind Tunnel (TWT) at the University of Notre Dame, and the Merrill Wind Tunnel (MWT) at California Institute of Technology. Wavefronts measurement were obtained using the Malley probe at sampling rates capable of resolving the range of frequencies at which the boundary layer is aerooptically active in each facility. Wavefront spectra for both heated walls and un-heated (baseline) cases were extracted and compared in order to investigate whether wall heating has a noticeable effect on the shape of wavefront spectra in the boundary layer, and thus the boundary layer dynamics. For all the data down to the lowest Reynolds number at which measurements were obtained at multiple wall temperatures, $\operatorname{Re}_{\theta}=4,200$, no significant changes in spectrum shape were observed. This result suggests that for $\operatorname{Re}_{\theta} \geq 4,200$, wall heating simply acts as a passive amplifier of boundary layer turbulence and does not alter the wall-normal integrated TBL statistics.

It was shown that for decreasing Reynolds number, the inertial range of aero-optic wavefront spectra is also reduced, which is consistent with other studies of the characteristics of turbulence spectra [17]. The empirical spectral model [14] was modified with the addition of an exponential decay term that approximates the experimentally observed high-frequency roll-off of wavefront spectra as a function of Reynolds number. The model was shown to be in good agreement with experimentally measured spectra. The scaling for the overall level of $O P D_{r m s}$, which was originally derived for high Reynolds number boundary layers, was shown to be also valid for low Reynolds numbers, $\operatorname{Re}_{\theta}>4,000$.

Measurements of $O P D_{r m s}$ for heated walls were also shown to be consistent with trends from previous experiments by Cress [12], and a method of estimating $O P D_{r m s}(\Delta T=0)$ from wavefront measurements of heatedwall boundary layers was demonstrated and discussed. This technique, along with the modification to the spectral model from [14] for Reynolds number dependence, should prove to be especially useful for applying optical techniques to experimentally study boundary layers at low subsonic speeds, as it allows for the problem of aerooptic 'invisibility' that occurs when freestream velocities enter the incompressible regime to be bypassed. Wall heating also poses a promising solution to the problem of low signal-to-noise ratios for thin turbulent boundary layers at higher Mach numbers. Both of these cases of wall-heating would allow for better experimental data for use in validating computational studies of aero-optics of turbulent boundary layers. The possibility of using aero-optic heated-wall TBL wavefront measurements to estimate skin friction non-intrusively was also explored, and the predictions were shown to be in good agreement with previous experimental results obtained at comparable Reynolds numbers.

\section{Acknowledgments}

This work is supported by the Air Force Office of Scientific Research, Grant number FA9550-12-1-0060. The U.S. Government is authorized to reproduce and distribute reprints for governmental purposes notwithstanding any copyright notation thereon. 


\section{References}

[1] Jumper, E.J., and Fitzgerald, E.J., 2001, "Recent Advances in Aero-Optics," Progress in Aerospace Sciences, 37, 299-339.

[2] Wang, M., Mani, A., and Gordeyev, S., "Physics and Computation of Aero-Optics", Annual Review of Fluid Mechanics, Vol. 44, pp. 299-321, 2012.

[3] Liepman, H.W., "Deflection and diffusion of a light ray passing through a boundary layer," Report SM-14397. Douglas Aircraft Company, Santa Monica Division, Santa Monica, CA, (1952).

[4] Sutton, G.W., "Effect of turbulent fluctuations in an optically active fluid medium." AIAA Journal, 7(9): 17371743, Sept. 1969.

[5] Tatarski, V.I., Wave Propagation in Turbulent Medium, New York: McGraw-Hill, 1961.

[6] Malley, M., Sutton, G. W. and Kincheloe, N., "Beam-Jitter Measurements of Turbulent Aero-Optical Path Length differences," Applied Optics, Vol. 31, pp. 4440-4443 (1992).

[7] Wang, K. and Wang, M., "Aero-optics of subsonic turbulent boundary layers," Journal of Fluid Mechanics, Vol. 696, pp. 122-151, 2012.

[8] Wyckham, C., and Smits, A., "Aero-Optic Distortion in Transonic and Hypersonic Turbulent Boundary Layers," AIAA Journal, Vol. 47, No. 9, pp. 2158-2168, 2009.

[9] Smith, A.E., Gordeyev, S., and Jumper, E.J., "Recent measurements of aero-optical effects caused by subsonic boundary layers," Opt. Eng, 52(7), 071404, 2013.

[10] Gordeyev, S., Jumper, E.J., Ng, T., and Cain, A., "Aero-Optical Characteristics of Compressible, Subsonic Turbulent Boundary Layer," AIAA Paper 2003-3606, 2003.

[11] Wittich, D. J., Gordeyev, S., and Jumper, E. J., "Revised scaling of optical distortions caused by compressible, subsonic turbulent boundary layers." AIAA Paper 2007-4009, 2007.

[12] Cress J., Gordeyev S., Post, M., and Jumper, E.J., "Aero-Optical Measurements in a Turbulent, Subsonic Boundary Layer at Different Elevation Angles," AIAA Paper 2008-4214, 2008.

[13] Gordeyev, S., Jumper, E.J., and Hayden, T., "Aero-Optics of Supersonic Boundary Layers," AIAA Journal, 50(3), 682-690, 2012.

[14] S. Gordeyev, A. E. Smith, J.A. Cress and E.J. Jumper, "Experimental studies of aero-optical properties of subsonic turbulent boundary layers," Journal of Fluid Mechanics, 740, pp 214-253, 2014.

[15] Cress, J., Optical Aberrations Cause by Coherent Structures in a Subsonic, Compressible, Turbulent Boundary Layer, Ph.D. thesis, University of Notre Dame, 2010.

[16] White, M. and Visbal, M., "Aero-optics of Compressible Boundary Layers in the Transonic Regime", AIAA Paper 2012-2984, 2012.

[17] Tatarski, V.I., The Effects of the Turbulent Atmosphere on Wave Propagation (trans. for NOAA by Israel Program for Scientific Translations, Jerusalem, 1971).

[18] York, D., Evensen, N., Martinez, M., and Delgado, J. "Unified equations for the slope, intercept, and standard errors of the best straight line." Am. J. Phys. 72 (3) March 2004.

[19] Hama, F.R., "Boundary-layer characteristics for smooth and rough surfaces, by Francis R. Hama," Trans. Soc. Nav. Arch. Mar. Eng. 62 (1954).

[20] Purtell, L.P., Klebanoff, P.S., and Buckley, F.T., "Turbulent boundary layer at low Reynolds number," Phys. Fluids 24(5), pp. 802-811, May 1981.

[21] Landweber, L., "The Frictional Resistance of Flat Plates at Zero Pressure Gradient," Trans. Soc. Nav. Arch. Mar. Eng. 61, pp. 5-32, 1953.

[22] Österlund, J.M., Experimental studies of zero pressure-gradient turbulent boundary layer flow, Ph.D. Thesis, Royal Institute of Technology, Stockholm, Sweden, 1999. 